\title{
Temperature Dependence of Crystallization Behavior in a Phase-Separated Blend of Poly( $\varepsilon$-caprolactone) Homopolymer and Poly( $\varepsilon$-caprolactone)-block-Polybutadiene Copolymer
}

\author{
Michiaki AKABA and Shuichi NOJIMA ${ }^{\dagger}$ \\ Department of Polymer Chemistry, Tokyo Institute of Technology, \\ H-125, 2-12-1 Ookayama, Meguro-ku, Tokyo 152-8552, Japan \\ (Received October 4, 2005; Accepted February 2, 2006; Published May 17, 2006)
}

\begin{abstract}
The crystallization behavior in a macroscopically phase-separated blend consisting of poly( $\varepsilon$-caprolactone) homopolymer (PCL) and PCL-block-polybutadiene copolymer (PCL- $b$-PB) is investigated both by synchrotron small-angle X-ray scattering (SR-SAXS) and polarized optical microscope (POM) as a function of crystallization temperature $T_{c}$. The characteristic size of the phase-separated structure $\xi$ existing in this blend before crystallization can arbitrarily be controlled by changing the phase separation time. When the blend with $\xi=2.5 \mu \mathrm{m}$ is quenched into various $T_{c}$, two scattering peaks are observed in the SR-SAXS curve during crystallization, which arise from the crystallized PCL and PCL- $b$-PB regions. The growth rates of these scattering peaks are identical at lower $T_{c}\left(\leq 35^{\circ} \mathrm{C}\right)$, while they deviate gradually with increasing $T_{c}$. Only one type of spherulites is observed for the blend with $\xi=10 \mu \mathrm{m}$ crystallized at $T_{c}=35^{\circ} \mathrm{C}$ to give a single growth rate, while two different types of spherulites appear at higher $T_{c}\left(\geq 40^{\circ} \mathrm{C}\right)$ and their growth rates are significantly different. Therefore the $T_{c}$ dependence of crystallization behavior observed by POM is qualitatively similar to that obtained by SR-SAXS, though $\xi$ is moderately different between both cases. [doi:10.1295/polymj.PJ2005148]

KEY WORDS Binary Blend / Phase-separated Structure / Crystallization / Small-angle X-ray Scattering / Optical Microscopy /
\end{abstract}

Morphology formation in polymer blends is sometimes driven by a combined effect of the liquid-liquid phase separation between components and the crystallization of constituent polymers. The mechanism of such morphology formation has been studied by many groups for crystalline/amorphous polymer blends with an UCST- or LCST-type phase-separated region. ${ }^{1-18}$ For example, we have previously investigated the crystallization process in a binary blend of poly( $\varepsilon$-caprolactone) homopolymer (PCL, crystalline) and polystyrene oligomer (PSO, amorphous) by timeresolved small-angle X-ray scattering with synchrotron radiation (SR-SAXS), ${ }^{2,6,7}$ where this blend had an UCST-type phase separation between PCL and PSO at the same temperature range of PCL crystallization. The process of morphology formation was considerably complicated, and the system consisted of two regions, amorphous region mainly made of PSO and crystallized region consisting of PCL lamellae and amorphous layers, at all times during morphology formation even for the blend without any phase separation before crystallization. In these studies, we could get SAXS peak from the crystallized region but not from the amorphous region since the crystallized region had a definite structure inside, and accordingly we obtained information on the morphology formation only from the crystallized region.
Therefore we believe that crystalline/crystalline polymer blends have a potential advantage to investigate the complicated morphology formation, because two kinds of crystallization behavior might be observed simultaneously to get more information on the morphology formation. However there are a limited number of studies on the morphology formation in crystalline/crystalline blends ${ }^{19-22}$ because two crystalline homopolymers are usually immiscible.

In a series of our recent studies, ${ }^{23,24}$ we used a binary blend consisting of poly ( $\varepsilon$-caprolactone) homopolymer (PCL) and PCL-block-polybutadiene copolymer (PCL- $b$-PB) by considering above points, where both polymers were crystalline and an UCST-type phase-separated region was situated in a same temperature range of crystallization. We have obtained following results; (1) two regions, crystallized PCL and PCL- $b$-PB regions, appeared during crystallization when the blend was directly quenched from the microphase-separated melt into crystallization temperature $T_{c}$ without any phase separation, where the crystallization rate of PCL was equal to that of PCL- $b$-PB and the overall rate of morphology formation was driven only by the crystallization of PCL chains (i.e., PCL and PCL blocks in PCL- $b$-PB). (2) When the blend was quenched into $T_{c}$ by passing through the UCST-type phase-separated region, the crystalli-

${ }^{\dagger}$ To whom correspondence should be addressed (Tel: +81-3-5734-2132, Fax: +81-3-5734-2888, E-mail: snojima@polymer.titech.ac.jp). 
zation rate of two polymers depended significantly on the characteristic size of the phase-separated structure $\xi$ existing in the blend before crystallization. That is, when $\xi<500 \mathrm{~nm}$ the crystallization rate of PCL was equal to that of PCL- $b$-PB, while when $\xi>500$ $\mathrm{nm}$ two rates deviated significantly and eventually they approached to the values of neat polymers with increasing $\xi$. We discussed the relation between $\xi$ and the crystal growth rate $D$ of PCL chains and pointed out that above experimental results obtained at a constant $T_{c}\left(=40^{\circ} \mathrm{C}\right)$ might change with changing $T_{c}$. This is because $D$ is critically dependent on $T_{c}$ and therefore the relation between $\xi$ and $D$ will change drastically at each $T_{c}$ to yield a different crystallization behavior.

In this study, we investigate the effect of $T_{c}$ on the crystallization behavior in a binary blend of PCL and PCL- $b-\mathrm{PB}$, where the phase separation between PCL and PCL- $b$-PB yields the heterogeneous structure with various $\xi$ prior to the crystallization, which can be controlled by the phase separation time. The crystallization process of this blend is observed both by SR-SAXS and polarized optical microscope (POM), which give information on the morphology at different sizes. The results obtained from a series of our studies, that is, the effects of $T_{c}$ as well as those of $\xi$ on the morphology formation in crystalline/crystalline binary blends, will contribute to the morphological design of these systems. That is, information during morphology formation will make it possible to control the crystallization behavior of each component and eventually final morphology formed in these polymer systems.

\section{EXPERIMENTAL}

\section{Polymers and Sample Preparation}

PCL $\left(M_{n}=3,500\right.$ and $\left.M_{w} / M_{n}=1.50\right)$ and PCL- $b$ PB $\left(M_{n}=13,000, M_{w} / M_{n}=1.16\right.$, and PCL:PB = 66:34 in volume ratio) used here are the same that we used in our previous study. ${ }^{23,24}$ This blend has an UCST-type phase separated region with a critical temperature at $c a .110^{\circ} \mathrm{C}$, and the melting temperature of PCL chains $T_{m}$ is $c a .60^{\circ} \mathrm{C}$ irrespective of composition. The melting temperatures meet with the cloud point curve at $\phi_{P C L}=0.2$ and 0.8 , where $\phi_{P C L}$ is the weight fraction of PCL in the blend, so that it is possible to develop the phase-separated structure with various $\xi$ by annealing the blends with $0.2<\phi_{P C L}<$ 0.8 at temperatures above $T_{m}$.

In this paper, we exclusively report on the SRSAXS and POM results for the blend with $\phi_{P C L}=$ 0.6 because they did not depend significantly on $\phi_{P C L}$. However, our previous results showed that the crystallization behavior of this blend depended intimately on $\xi{ }^{24}$ That is, the crystallization rates of PCL and PCL$b$-PB were identical at $40^{\circ} \mathrm{C}$ for the blend with $\xi \leq$ $500 \mathrm{~nm}$, while they deviated gradually with increasing $\xi$. For example, the crystallization rate of PCL- $b-\mathrm{PB}$ was two-third of that of PCL for the blend with $\xi=2.5 \mu \mathrm{m}$. Therefore we chose $\xi=2.5 \mu \mathrm{m}$ for investigating the $T_{c}$ dependence of crystallization behavior by SR-SAXS. In addition, we chose $\xi=10 \mu \mathrm{m}$ as well as $2.5 \mu \mathrm{m}$ for the spherulite observation by POM because we could not observe the separate crystallization of PCL and PCL- $b$-PB for the blend with $\xi=2.5 \mu \mathrm{m}$.

First the blend was quenched from the microphaseseparated melt at $120^{\circ} \mathrm{C}$ into $70^{\circ} \mathrm{C}\left(>T_{m}\right)$ and annealed there for $42 \mathrm{~h}$ (corresponding to $\xi=2.5 \mu \mathrm{m}$ ) or $168 \mathrm{~h}(\xi=10 \mu \mathrm{m})$ to yield two kinds of phase-separated structure before crystallization. $\xi$ was evaluated by the relation $\xi \propto t^{0.83}$ previously obtained for this blend, ${ }^{24}$ because a small difference in refractive index between PCL and PCL- $b$-PB led to the unreliable $\xi$ when it was estimated by an optical microscope.

\section{SR-SAXS Measurements}

The SR-SAXS measurement was performed at Photon Factory in High-Energy Accelerator Research Organization, Tsukuba Japan, with a small-angle Xray equipment for solution (SAXES) installed at beam line BL-10C. Details of the optics and the instrumentation were described elsewhere. ${ }^{25,26}$ The SR-SAXS measurements were continued until the integrated intensity did not change any more, and each time-sliced SAXS intensity during $10 \mathrm{sec}$ was obtained as a function of wave number $s\left(=\frac{2}{\lambda} \sin \theta ; 2 \theta\right.$ : scattering angle, $\lambda$ : X-ray wave length $(=0.1488 \mathrm{~nm}))$ after correcting background scattering. The SAXS curves thus obtained were very complicated because they consisted of three scattering peaks and also an increasing intensity toward $s \rightarrow 0$ (see Figure 1). To evaluate the angular position and intensity of each scattering peak accurately, we used the Lorentz function ${ }^{27}$ for each scattering peak and the Debye-Bueche equation for the increasing intensity at lower $s,{ }^{6,7}$ as we described in our previous manuscript. ${ }^{23}$ The peak position (or spacing) and peak intensity were finally evaluated as a function of crystallization time $t_{c}$ at each $T_{c}$.

\section{POM Observations}

The macroscopic change in morphology by the crystallization of PCL chains was observed at various $T_{c}$ by a polarized optical microscope (Olympus BX50) with a temperature-controlled hot stage (Linkam LTS-350). ${ }^{28}$ The morphological change was monitored between crossed polarizers, and the average radius of spherulites was measured at each $T_{c}$ as a function of $t_{c}$ to evaluate the growth rate of spherulites 


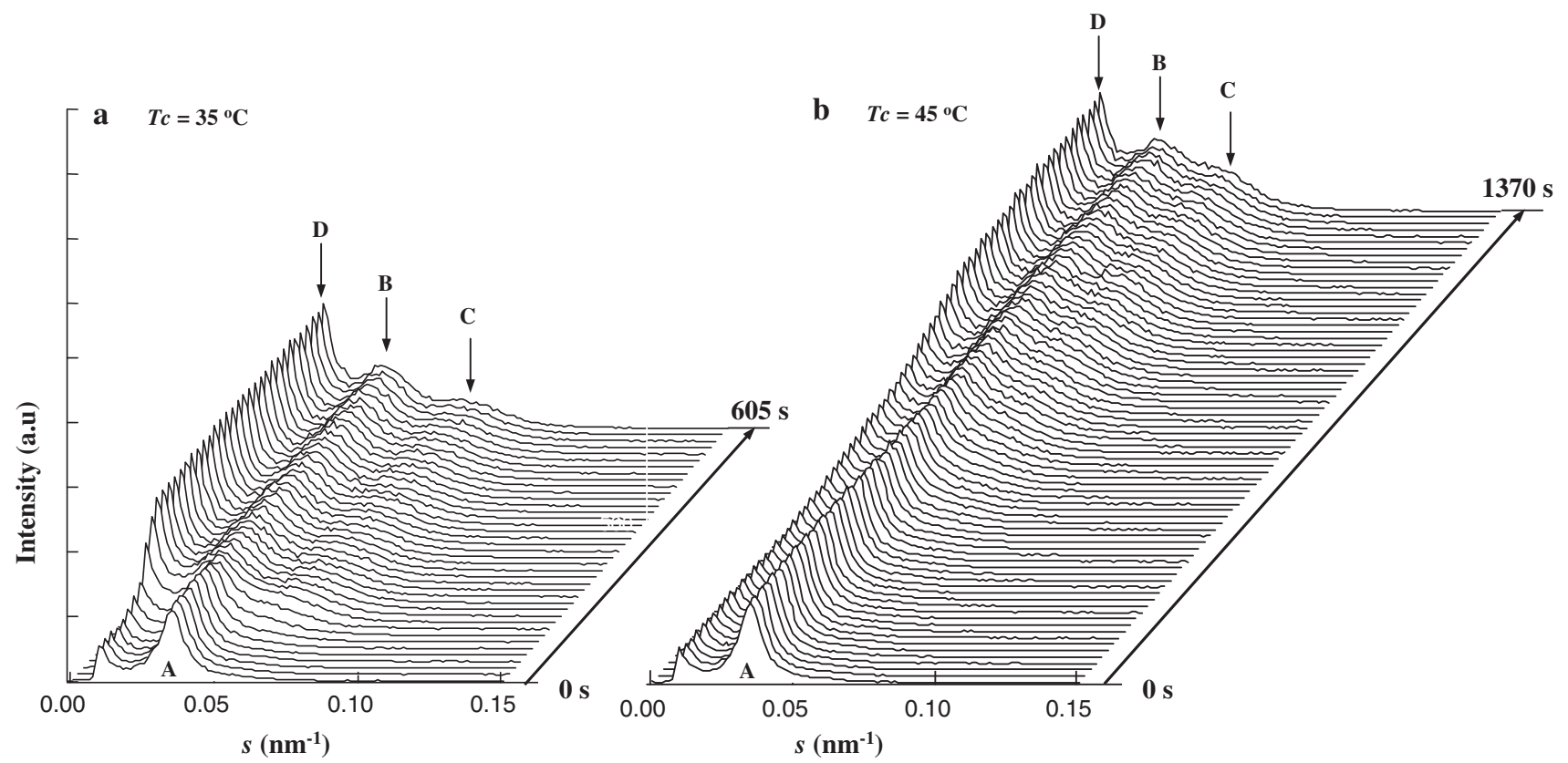

Figure 1. SR-SAXS curves during crystallization of PCL chains at $35^{\circ} \mathrm{C}$ (a) and $45^{\circ} \mathrm{C}$ (b) for the macroscopically phase-separated blend with $\xi=2.5 \mu \mathrm{m}$.

appearing in the blend. Here, we used the blends with $\xi=2.5 \mu \mathrm{m}$ and $10 \mu \mathrm{m}$ because the difference in $\xi$ yielded a distinct difference in the spherulite growth behavior.

\section{RESULTS AND DISCUSSION}

\section{Overview of Crystallization Process Measured by SR-SAXS}

Figure 1 shows time-resolved SR-SAXS curves for the phase-separated blend with $\xi=2.5 \mu \mathrm{m}$ crystallized at $35^{\circ} \mathrm{C}$ (a) and $45^{\circ} \mathrm{C}$ (b). These SR-SAXS curves are virtually similar in shape to those previously reported for the blends without any phase separation. ${ }^{23}$ That is, the scattering peak (denoted by A), which arises from the microdomain structure in the melt, decreases in intensity with increasing $t_{c}$ and simultaneously two scattering peaks appear at lower (B) and higher (C) angles, which come from the crystallized PCL- $b$-PB and PCL regions, respectively. In addition, we can observe a monotonically increasing scattering toward $s \rightarrow 0$ (D), which is ascribed to the mosaic structure consisting of the crystallized PCL- $b$-PB and PCL regions. The qualitative comparison between Figures $1 \mathrm{a}$ and $1 \mathrm{~b}$ indicates that the crystallization process is merely more prolonged at higher $T_{c}$ without any substantial difference.

The $t_{c}$ dependence of the spacing (i.e., the alternating period of lamellar morphology formed in PCL and PCL- $b$-PB regions) and the normalized intensity (i.e., the peak intensity divided by the maximum intensity) evaluated from each scattering peak is plotted in
Figure 2 for the phase-separated blend with $\xi=2.5$ $\mu \mathrm{m}$ crystallized at $T_{c}=35^{\circ} \mathrm{C}(\mathrm{a}, \mathrm{d}), 40^{\circ} \mathrm{C}(\mathrm{b}, \mathrm{e})$, and $45^{\circ} \mathrm{C}$ (c, f). The spacing from the microdomain structure is gradually replaced with two kinds of spacing, one from the lamellar morphology formed in the crystallized PCL- $b$-PB region and the other from the crystallized PCL region, at the middle of $t_{c}$, which is similar to that for the blend without any phase separation. ${ }^{23}$ However, the $t_{c}$ dependence of the normalized intensity is not similar to that for the blend without phase separation. That is, the normalized intensities from two regions make one master curve at $T_{c}=35^{\circ} \mathrm{C}$ (Figure 2d) while they deviate significantly at $T_{c}=40$ and $45^{\circ} \mathrm{C}$ (Figures 2e and 2f), indicating that the crystallization rates of PCL and PCL- $b$ PB are equivalent at $T_{c}=35^{\circ} \mathrm{C}$ but they differ considerably at $T_{c} \geq 40^{\circ} \mathrm{C}$. We showed previously that the crystallization behavior at $T_{c}=40^{\circ} \mathrm{C}$ was intimately dependent on $\xi$; the crystallization rate of PCL was equal to that of PCL- $b$-PB for the blend with a small $\xi(\leq 500 \mathrm{~nm})$ but not for the blend with a large $\xi(>500 \mathrm{~nm})$. Figures $2 \mathrm{~d}-2 \mathrm{f}$ indicate that even if $\xi$ is enough large $(=2.5 \mu \mathrm{m})$ the crystallization behavior is also critically dependent on $T_{c}$.

\section{$T_{c}$ Dependence of Crystallization Rate}

It is easily found from Figures $2 a-2 c$ that the spacing of lamellar morphology formed in PCL- $b$-PB and PCL regions is constant irrespective of $T_{c}$, and is plotted in Figure 3 against $T_{c}$. The spacing of the PCL lamellar morphology is $15.0 \mathrm{~nm}$ and comparable to that of neat PCL $(14.0 \mathrm{~nm}$ when crystallized at 

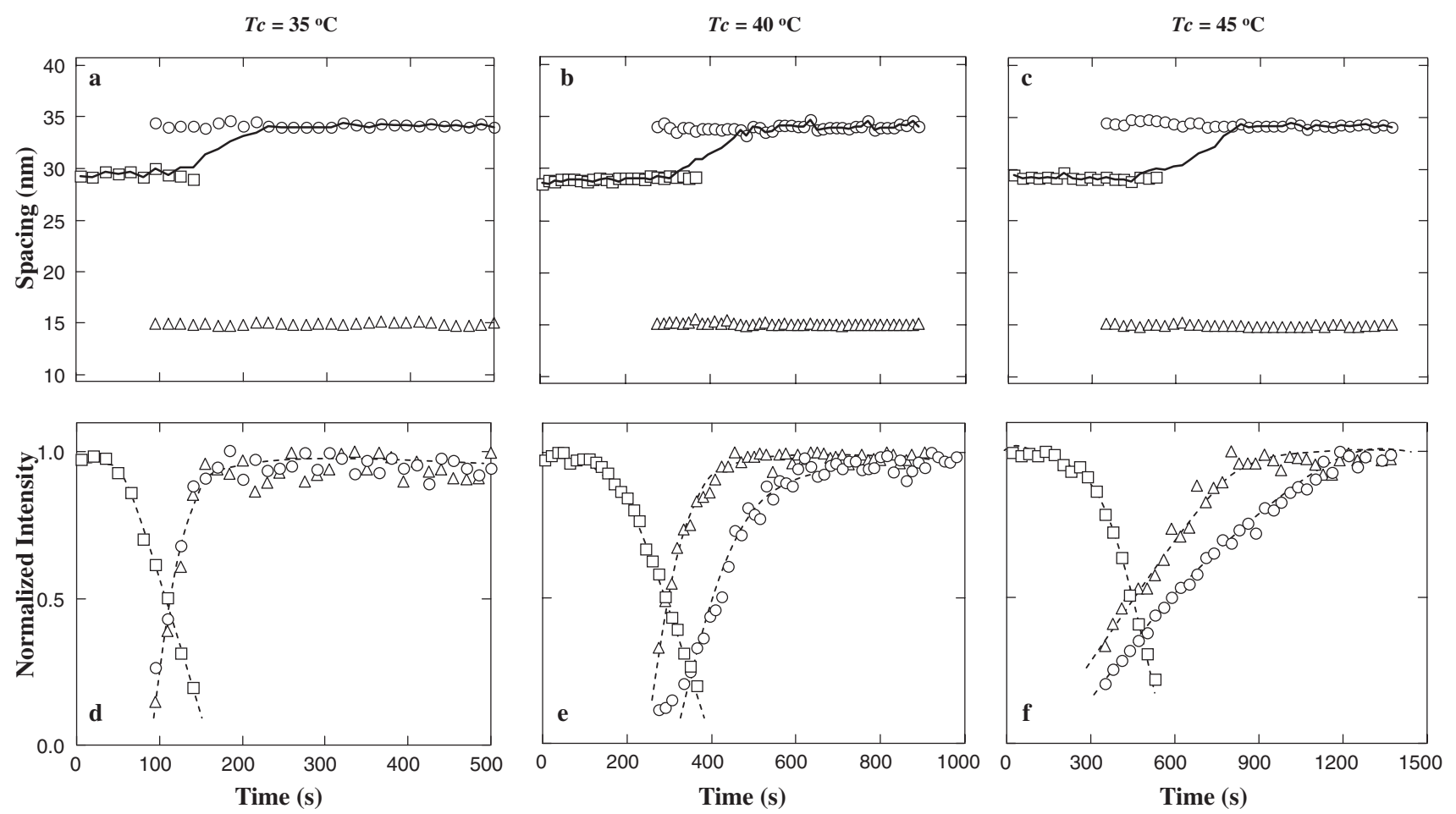

Figure 2. $t_{c}$ dependence of the spacing $(\mathrm{a}-\mathrm{c})$ and normalized peak intensity $(\mathrm{d}-\mathrm{f})$ evaluated from SR-SAXS peaks arising from the microdomain structure $(\square)$, crystallized PCL- $b$-PB region $(\bigcirc)$, and crystallized PCL region $(\triangle)$ during crystallization at $35^{\circ} \mathrm{C}($ left), $40^{\circ} \mathrm{C}$ (middle), and $45^{\circ} \mathrm{C}$ (right) for the phase-separated blend with $\xi=2.5 \mu \mathrm{m}$. The solid curves in panels a-c represent the spacing when the scattering peaks from the microdomain structure and crystallized PCL- $b$-PB region are approximated by a single Lorentz function.

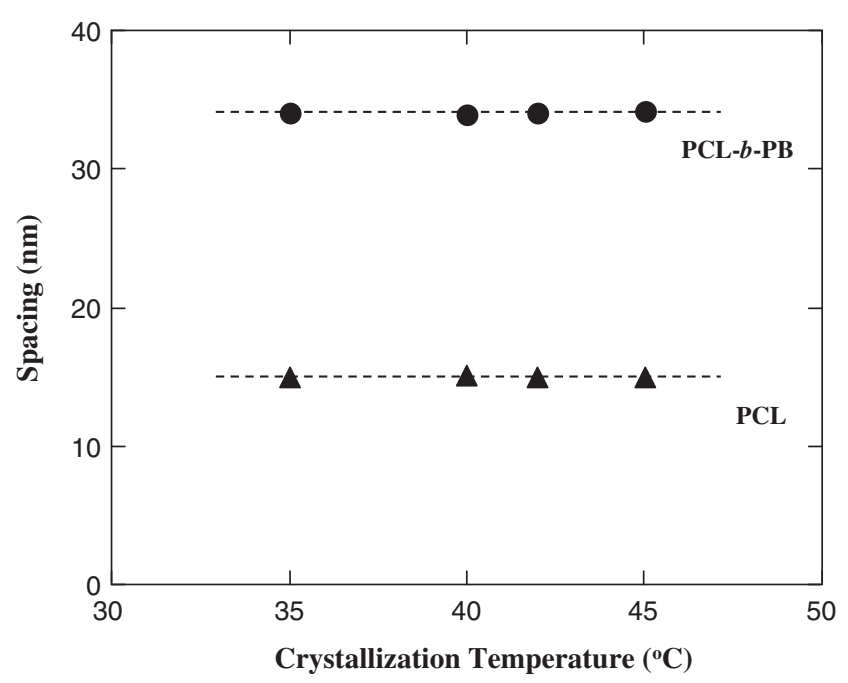

Figure 3. $T_{c}$ dependence of the spacing of the lamellar morphology formed in the crystallized PCL- $b$-PB $(\bullet)$ and PCL $(\boldsymbol{\Delta})$ regions for the phase-separated blend with $\xi=2.5 \mu \mathrm{m}$.

$40^{\circ} \mathrm{C}$ ), indicating that the PCL lamellar morphology is almost made up of pure PCL. However, the spacing of the PCL- $b$-PB lamellar morphology is $34.5 \mathrm{~nm}$ and moderately larger than that of neat PCL- $b$-PB $\left(29.3 \mathrm{~nm}\right.$ at $\left.40^{\circ} \mathrm{C}\right)$, suggesting that some amount of PCL is incorporated in the PCL- $b$-PB lamellar morphology to yield the larger spacing. The theoretical phase diagram for binary blends with phase separation and crystallization in a same temperature range predicts that the crystallization of PCL chains yields pure PCL- $b$-PB and PCL phases in equilibrium, ${ }^{29}$ and therefore the binodal curve does not intervene any more in the morphology formation under the melting temperature of PCL chains. The significantly large spacing for the PCL- $b$-PB lamellar morphology suggests the non-equilibrium crystallization, for which we will discuss later in relation to the spherulite structure.

Figure 4 shows the $T_{c}$ dependence of the half-time of crystallization $t_{1 / 2}$, a measure of the crystallization rate of constituent polymers in each region, for the phase-separated blend with $\xi=2.5 \mu \mathrm{m}$, where $t_{1 / 2}$ is evaluated from the plot of the normalized peak intensity against $t_{c}$ (Figures $2 \mathrm{~d}-2 \mathrm{f}$ ). It is clearly found that at $T_{c}=35^{\circ} \mathrm{C}$ the values of $t_{1 / 2}$ for PCL- $b$-PB and PCL are identical but they deviate significantly with increasing $T_{c}$. In addition, $t_{1 / 2}$ for the quenched blend with a small $\xi$, where $t_{1 / 2}$ for PCL is equal to that of PCL- $b$-PB at any $T_{c}$, is also plotted in Figure 4, in which $t_{1 / 2}$ for the quenched blend takes a middle position between those for PCL and PCL- $b$-PB in the phase-separated blend. This is a new finding of the present study and definitely shows that the combination of $\xi$ and $T_{c}$ drives the crystallization behavior of this blend. That is, increasing $T_{c}$ at constant $\xi$ leads to the separate crystallization of PCL and PCL- $b$-PB, 


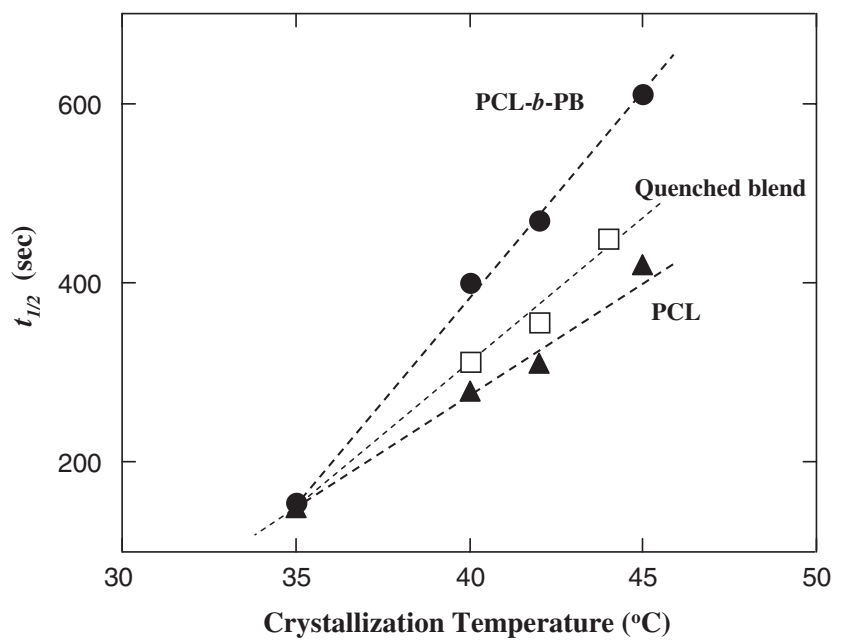

Figure 4. $T_{c}$ dependence of the half-time of crystallization $t_{1 / 2}$ for PCL- $b$-PB $(\bullet)$ and PCL $(\boldsymbol{\Delta})$ in the phase-separated blend with $\xi=2.5 \mu \mathrm{m}$. $\square$ represents the result for the quenched blend, where $t_{1 / 2}$ is equivalent for PCL and PCL- $b$-PB.

and therefore $T_{c}$ has the same effect as $\xi$ on the crystallization behavior of two polymers.

\section{Overview of Crystallization Process Observed by POM}

Figure 5 shows the typical spherulites observed during crystallization at $35^{\circ} \mathrm{C}(\mathrm{a}, \mathrm{d}), 40^{\circ} \mathrm{C}(\mathrm{b}, \mathrm{e})$, and $45^{\circ} \mathrm{C}(\mathrm{c}, \mathrm{f})$ for the phase-separated blend with $\xi=2.5 \mu \mathrm{m}$ (upper panels) and $\xi=10 \mu \mathrm{m}$ (lower panels). The spherulites shown in panels a-d are all classified into a same type judging from their appearances, which is similar to the spherulite observed in neat PCL (Figure 6a). However, two different spherulites are formed for the blend with $\xi=10 \mu \mathrm{m}$ crystallized at $T_{c}=40$ and $45^{\circ} \mathrm{C}(\mathrm{e}, \mathrm{f})$, that is, one is similar to that mentioned above (denoted by $\mathrm{A}$ in panels e and f) and the other that with a distinct banded structure (B), which is analogous to the spherulite observed for neat PCL- $b$-PB (Figure 6b). Therefore it is easily speculated that the spherulite denoted by A or B in Figures $5 \mathrm{e}$ and $5 \mathrm{f}$ corresponds to that appearing in neat PCL (Figure 6a) or PCL- $b$-PB (Figure 6b), respectively, and consequently the spherulite $\mathrm{A}$ is mainly composed of PCL while the spherulite B of PCL- $b$-PB.

The spherulites with the banded structure are frequently reported for completely miscible blends consisting of crystalline/amorphous homopolymers ${ }^{30-33}$ and microphase-separated crystalline-amorphous diblock copolymers. ${ }^{34,35}$ In particular, PCL- $b$-PB forms regularly banded spherulites at any composition and $T_{c},{ }^{34}$ in which amorphous PB blocks covalently linked with PCL blocks can intimately interact with PCL blocks during crystallization though the repulsive interaction works between PCL and PB blocks. Therefore it is supposed that the interaction between amor-
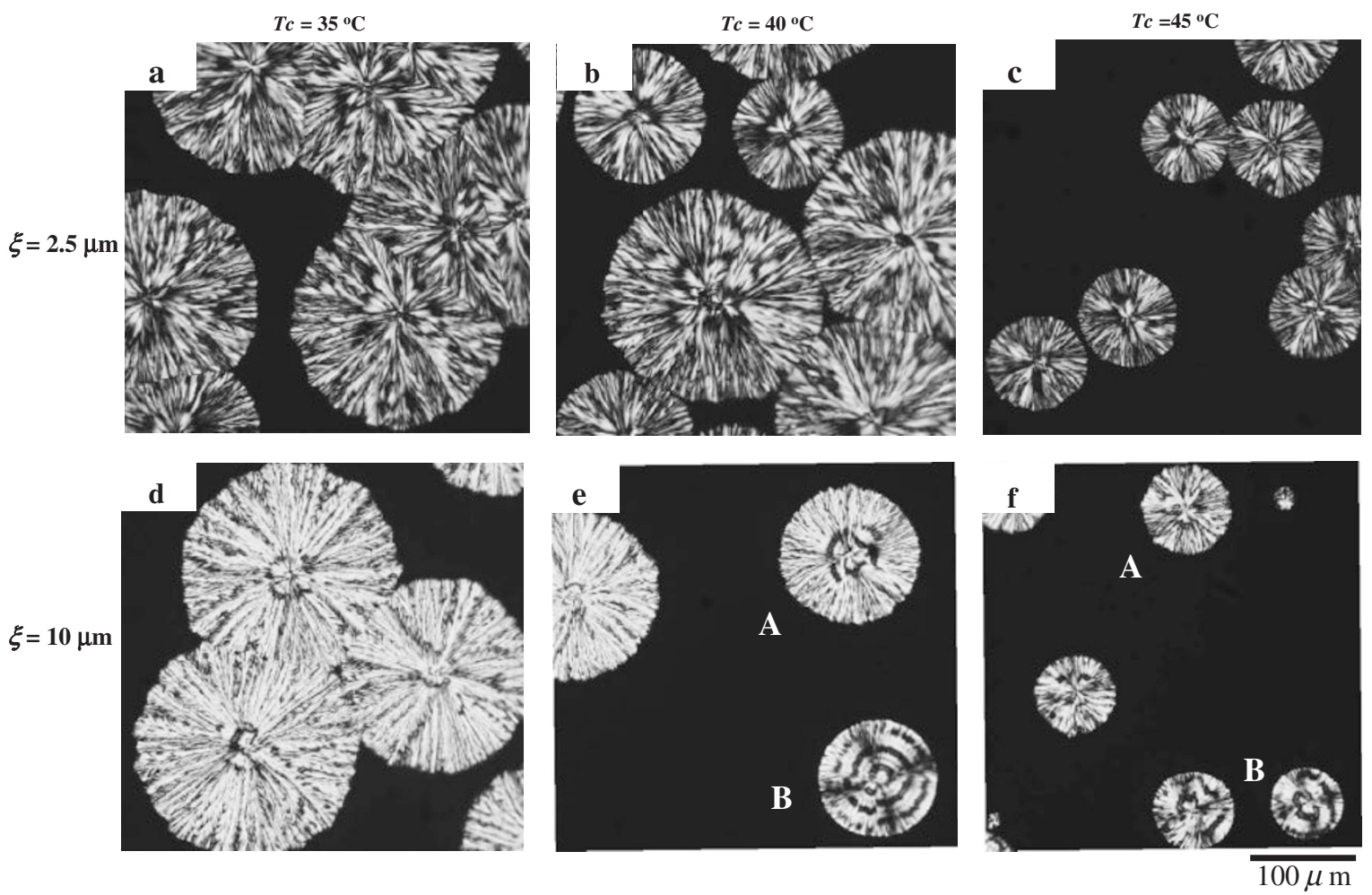

Figure 5. Typical spherulites observed during crystallization at each $T_{c}$ for the phase-separated blend with $\xi=2.5 \mu \mathrm{m}(\mathrm{a}-\mathrm{c})$ and $\xi=10 \mu \mathrm{m}(\mathrm{d}-\mathrm{f})$. Two different types of spherulites (indicated by A and B in panels e and f) can be observed only for the blend with $\xi=10 \mu \mathrm{m}$ crystallized at 40 and $45^{\circ} \mathrm{C}$. 

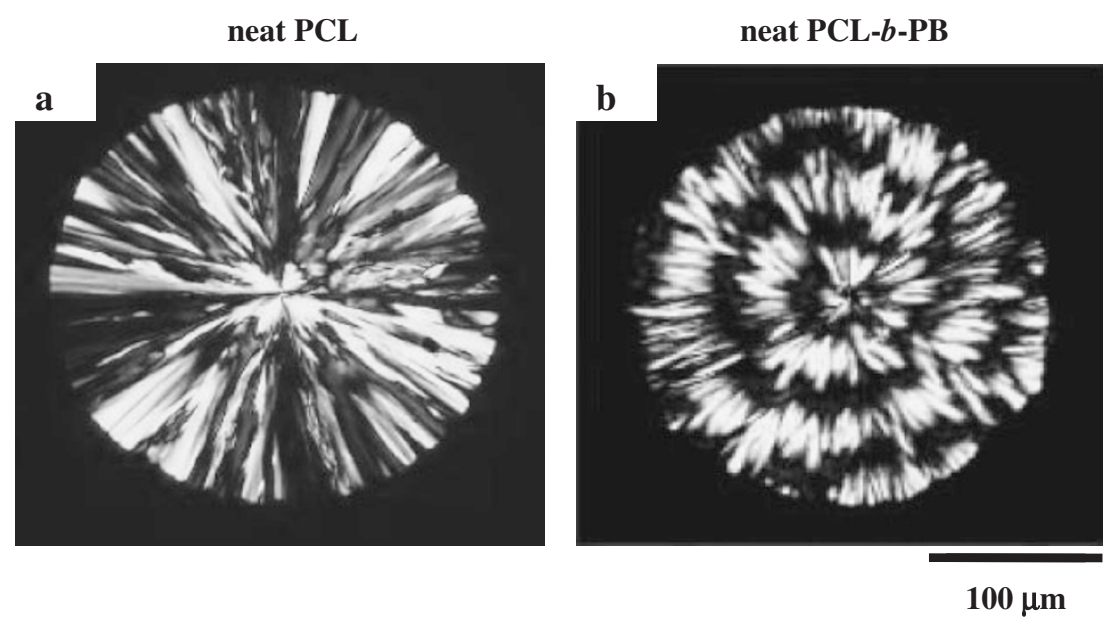

Figure 6. Typical spherulites observed for neat PCL (a) and PCL- $b$-PB (b) crystallized at $40{ }^{\circ} \mathrm{C}$.
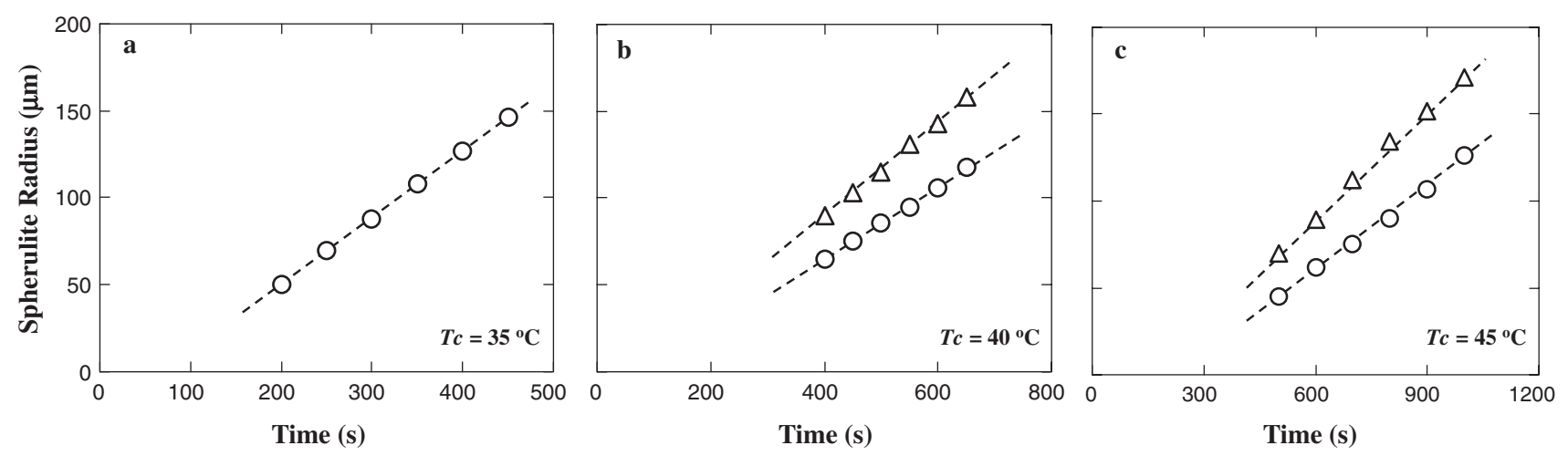

Figure 7. Spherulite growth rate in the phase-separated blend with $\xi=10 \mu \mathrm{m}$ crystallized at each $T_{c}$ indicated. $\triangle$ and $\bigcirc$ correspond to the growth rate of spherulites $\mathrm{A}$ and $\mathrm{B}$, respectively, in Figures $5 \mathrm{e}$ and $5 \mathrm{f}$.

phous and crystalline chains is necessary during crystallization to form such banded spherulites, because completely immiscible crystalline/amorphous blends never form such banded spherulites. However, the detailed mechanism to form banded spherulites is not fully understood.

It is puzzling to consider that the SAXS results show two different kinds of crystallization occurring in the system while the POM observation indicates a single spherulite growth for the sample with $\xi=2.5$ $\mathrm{nm}$, as shown in Figures 4 and 5. One plausible interpretation for this contradictory result is that the PCL crystallization is significantly decelerated by the presence of amorphous PCL- $b$-PB, but it controls the total structure and growth rate of the spherulite, and eventually the crystallization of PCL- $b$-PB follows with a slightly slow rate. It is necessary to quantitatively elucidate the relationship between the crystallization rates on different sizes.

\section{$T_{c}$ Dependence of Spherulite Growth Rate}

The average radius of spherulites evaluated from Figures $5 \mathrm{~d}-5 \mathrm{f}$ is plotted in Figure 7 against $t_{c}$ for var- ious $T_{c}$, which makes a line in every case. As previously mentioned, we have only one type of spherulites at $T_{c}=35^{\circ} \mathrm{C}$, so that we obtain one line (Figure 7a), while we have two types of spherulites at $T_{c}=40$ and $45^{\circ} \mathrm{C}$ whose growth rates are significantly different, so that we have two lines (Figures $7 b$ and $7 c$ ). The growth rate of PCL spherulites is distinctly faster than that of PCL- $b$-PB spherulites. This fact is qualitatively consistent with the SR-SAXS results shown in Figures $2 \mathrm{e}$ and $2 \mathrm{f}$, where the scattering peak from the PCL lamellar morphology appears first followed by that from the PCL- $b$-PB lamellar morphology at $T_{c}=40$ and $45^{\circ} \mathrm{C}$ and the time lag increases with increasing $T_{c}$, though $\xi$ in Figure 2 is moderately smaller than that in Figure 7.

The spherulite growth rate $G$ can be evaluated from the slope of these lines shown in Figure 7, and is plotted in Figure 8 against $T_{c}$. The values of $G$ for PCL and PCL- $b$-PB spherulites increase with decreasing $T_{c}$ and finally they coincide at $T_{c}=35^{\circ} \mathrm{C}$. Figure 8 also contains $G$ for the quenched blend with small $\xi$ $(\ll 100 \mathrm{~nm})$, which has intermediate values between $G$ of PCL and PCL- $b$-PB spherulites for the phase- 


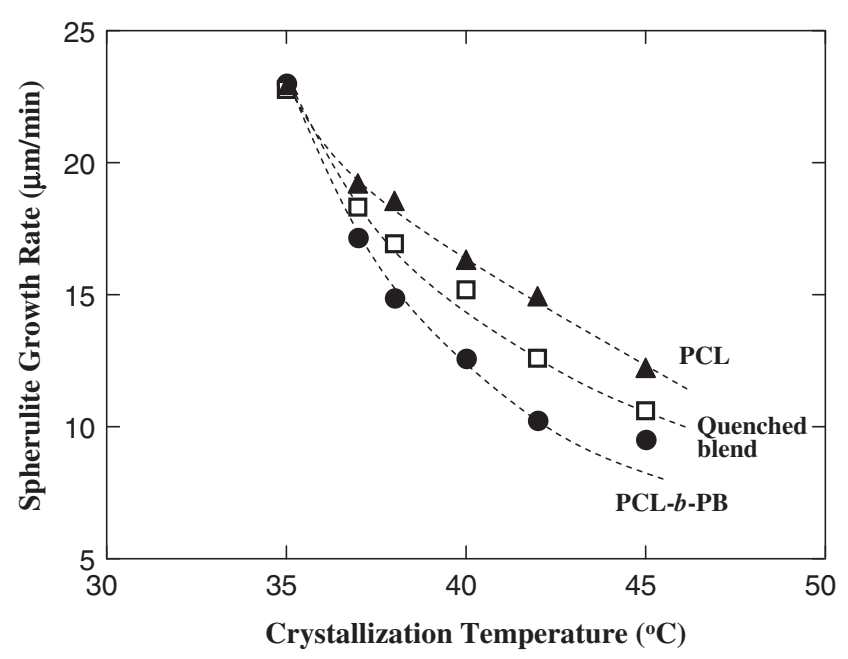

Figure 8. $T_{c}$ dependence of the growth rate of each spherulite in the phase-separated blend with $\xi=10 \mu \mathrm{m}(\boldsymbol{\bullet}$ and $\mathbf{\Delta})$. $\square$ represents the result in the quenched blend, where one type of spherulites can be observed.

separated blend. Figure 8 indicates that $T_{c}$ affects the crystallization behavior of our blend in the same way as $\xi$, and it leads to exactly the same conclusion obtained by SR-SAXS measurements (Figure 4). That is, the inverse of $t_{1 / 2}$, which is a measure of the crystallization rate obtained from SR-SAXS measurements, decreases monotonically with increasing $T_{c}$, though there is a large difference in the decreasing behavior between two results; $t_{1 / 2}^{-1}$ reduces faster than that of $G$. This is because there is a large difference in the structural size from which we get information on the crystallization behavior; $t_{1 / 2}^{-1}$ is based on the lamellar morphology and $G$ on the spherulite. Therefore, the growth mechanism of crystals on the lamella size and also on the spherulite size should be clarified for the quantitative comparison of both the results. In summary, PCL and PCL- $b$-PB make the separate crystallization with increasing $T_{c}$, which gives the same effect with increasing $\xi$ at constant $T_{c}$, as demonstrated in our previous study. ${ }^{24}$

\section{Detailed Analysis of Spherulite Structure}

The POM results show the difference in crystallization rate at larger $\xi$ than SR-SAXS results; two distinctly different spherulites are not observed up to $\xi=10 \mu \mathrm{m}$ while SR-SAXS results reveal the difference in crystallization rate at $\xi=2.5 \mu \mathrm{m}$. It is more difficult to find out the difference in the spherulite structure because the appearance within spherulites changes slightly and continuously with changing $\xi$, and even if there is a difference in the spherulite structure at smaller $\xi$ we might fail to notice it. Therefore, the SR-SAXS results are more reliable and sensitive for detecting the difference in crystallization rate of two regions. However, the POM observation contains

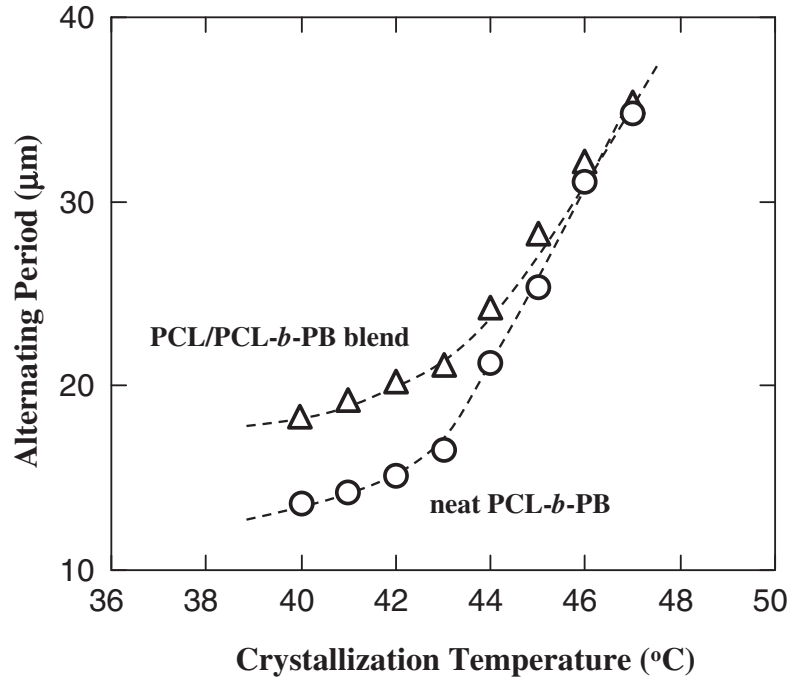

Figure 9. $T_{c}$ dependence of the alternating period of banded spherulites appearing in the phase-separated blend with $\xi=10$ $\mathrm{mm}(\triangle)$ and neat PCL- $b$-PB $(\bigcirc)$.

another information on the morphology formed in this blend.

Figure 9 shows the $T_{c}$ dependence of the alternating period of banded spherulites shown in Figures 5e and $5 f$, together with that formed in neat PCL- $b$-PB. It is phenomenologically known from our previous study ${ }^{34}$ that the alternating period depends significantly on the volume fraction of PCL chains (i.e., PCL and PCL blocks in PCL- $b$-PB) in the blend $\phi_{P C L}^{\prime}$ as well as $T_{c}$. That is, the period increases with increasing $\phi_{P C L}^{\prime}$ and $T_{c}$, though the mechanism is unknown. Figure 9 shows that at lower $T_{c}\left(\leq 45^{\circ} \mathrm{C}\right)$ the period in the blend is larger than that in neat PCL- $b-\mathrm{PB}$, suggesting that the PCL- $b$-PB region contains some amount of PCL and eventually $\phi_{P C L}^{\prime}$ is slightly larger than that of neat PCL- $b$-PB to give the larger period. This fact is consistent with the result obtained from Figure 3, where the spacing of the PCL- $b$-PB lamellar morphology in the blend is significantly larger than that of neat PCL- $b$-PB. At higher $T_{c}\left(\geq 45^{\circ} \mathrm{C}\right)$, on the other hand, two periods merge into one, suggesting that $\phi_{P C L}^{\prime}$ in the PCL- $b$-PB region is equivalent to that in neat PCL- $b$-PB and therefore the PCL- $b$-PB region is made up of pure PCL- $b$-PB without any PCL because the crystallization of PCL blocks is so slow for PCL to be completely rejected from the crystallized PCL- $b$ $\mathrm{PB}$ region.

As mentioned above, it is difficult to directly compare the results obtained by SR-SAXS and POM. However, the SR-SAXS method is advantageous to precisely find the difference in crystallization behavior between two regions, and the POM observation, in particular, the banded spherulite provides another information on the lamellar morphology formed in two regions during crystallization. 


\section{CONCLUSIONS}

We have studied the crystallization behavior of PCL chains in a macroscopically phase-separated blend consisting of PCL and PCL- $b$-PB as a function of $T_{c}$ both by time-resolved SR-SAXS and POM. This blend has an UCST-type phase-separated region in the crystallization range of PCL chains, and it is possible to control the phase-separated structure with various $\xi$ prior to the crystallization, from which we can start the crystallization by quenching the blend into various $T_{c}$. The following results were obtained from this study.

1) SR-SAXS measurements revealed that the crystallization rates of PCL and PCL- $b$-PB were significantly different at higher $T_{c}\left(\geq 40^{\circ} \mathrm{C}\right)$ for the blend with $\xi=2.5 \mu \mathrm{m}$, while they crystallized simultaneously at $T_{c}=35^{\circ} \mathrm{C}$. Because these crystallization rates were identical even at $T_{c}=40$ ${ }^{\circ} \mathrm{C}$ for the blend with smaller $\xi(\leq 500 \mathrm{~nm}$ ) as previously reported, it was concluded that decreasing $T_{c}$ has a same effect with decreasing $\xi$ on the crystallization behavior of PCL chains.

2) When $\xi=2.5 \mu \mathrm{m}$ we observed only one type of spherulites and eventually a single spherulite growth rate at any $T_{c}$, while when $\xi=10 \mu \mathrm{m}$ two different types of spherulites were observed at higher $T_{c}\left(\geq 40^{\circ} \mathrm{C}\right)$, whose growth rates were significantly different. Therefore, the effect of $T_{c}$ on the crystallization behavior observed by POM was consistent with that obtained by SR-SAXS though $\xi$ was moderately different in both cases.

Above facts clearly indicate that the crystallization behavior of this blend is driven by the interplay between the phase-separated structure before crystallization (or $\xi$ ) and the crystal growth rate $D$ (or $T_{c}$ ). Therefore it is necessary for the morphological control of binary blends with phase separation and crystallization exiting in a same temperature range to understand the detailed relationship between $\xi$ and $D$ (or $T_{c}$ ).

Acknowledgment. This study was partially supported by NEDO (New Energy and Industrial Technology Development Organization) launched in 2001 and also by Grand-in-Aid for Scientific Research (No. 17350102) from the Ministry of Education, Science, Sports, and Culture of Japan. The SR-SAXS measurement has been performed under the approval of Photon Factory Advisory Committee (No. 2004G093).

\section{REFERENCES}

1. Y. Hirata and T. Kotaka, Polym. J., 13, 272 (1981).

2. S. Nojima, Y. Terashima, and T. Ashida, Polymer, 27, 1007 (1986).
3. N. Inaba, K. Sato, S. Suzuki, and T. Hashimoto, Macromolecules, 19, 1690 (1986).

4. R. M. Briber and F. Khoury, Polymer, 28, 38 (1987).

5. H. Tanaka and T. Nishi, Phys. Rev., 39, 783 (1989).

6. S. Nojima, K. Satoh, and T. Ashida, Macromolecules, 24, 942 (1991).

7. S. Nojima, K. Kato, M. Ono, and T. Ashida, Macromolecules, 25, 1922 (1992).

8. N. Kinami, T. Okuyama, M. Okamoto, and T. Inoue, Polymer, 36, 4449 (1995).

9. R. L. Morgan, M. J. Hill, P. J. Barham, and C. J. Frye, Polymer, 38, 1903 (1997).

10. H. L. Chen and M. S. Hsiao, Macromolecules, 31, 6579 (1998).

11. H. L. Chen, J. C. Hwang, J. M. Yang, and R. C. Wang, Polymer, 39, 6983 (1998).

12. H. M. Shabana, R. H. Olley, D. C. Bassett, and B. J. Jungnickel, Polymer, 41, 5513 (2000).

13. J. K. Kim, B. K. Kim, and M. Park, J. Polym. Sci., Part B: Polym. Phys., 38, 707 (2000).

14. J. K. Lee, W. S. Choi, Y. K. Kwon, and K. H. Lee, Polymer, 43, 2827 (2002).

15. G. Matsuba, K. Shimizu, H. Wang, Z. Wang, and C. C. Han, Polymer, 44, 7459 (2003).

16. M. Tsuburaya and H. Saito, Polymer, 45, 1027 (2004).

17. G. Matsuba, K. Shimizu, H. Wang, Z. Wang, and C. C. Han, Polymer, 45, 5137 (2004).

18. K. Shimizu, H. Wang, Z. Wang, G. Matsuba, H. Kim, and C. C. Han, Polymer, 45, 7061 (2004).

19. J. P. Penning and R. St. J. Manley, Macromolecules, 29, 84 (1996).

20. L. Z. Liu, B. Chu, J. P. Penning, and R. St. J. Manley, Macromolecules, 30, 4398 (1997).

21. J. C. Lee, H. Tazawa, T. Ikehara, and T. Nishi, Polym. J., 30, 780 (1998).

22. Z. Qiu, S. Fujinami, M. Komura, K. Nakajima, T. Ikehara, and T. Nishi, Polym. J., 36, 642 (2004).

23. M. Akaba and S. Nojima, Polym. J., 37, 464 (2005).

24. M. Akaba and S. Nojima, Polym. J., 37, 584 (2005).

25. S. Nojima, K. Kato, S. Yamamoto, and T. Ashida, Macromolecules, 25, 2237 (1992).

26. S. Nojima, N. Kikuchi, A. Rohadi, S. Tanimoto, and S. Sasaki, Macromolecules, 32, 3727 (1999).

27. G. Will, M. Bellotto, W. Parrish, and M. Hart, J. Appl. Crystallogr., 21, 182 (1988).

28. S. Nojima, Y. Akutsu, M. Akaba, and S. Tanimoto, Polymer, 46, 4060 (2005).

29. W. R. Burghardt, Macromolecules, 22, 2482 (1989).

30. F. B. Khambatta, F. Warner, T. Russell, and R. S. Stein, J. Polym. Sci., Polym. Phys. Ed., 14, 1391 (1976).

31. S. Nojima, K. Watanabe, Z. Zheng, and T. Ashida, Polym. J., 20, 823 (1988).

32. W. Li, R. Yan, and B. Jiang, Polymer, 33, 889 (1992).

33. J. Xu, B. H. Guo, J. J. Zhou, L. Li, J. Wu, and M. Kowalczuk, Polymer, 46, 9716 (2005).

34. S. Nojima, D. Wang, and T. Ashida, Polym. J., 23, 1473 (1991).

35. T. Shiomi, K. Imai, K. Takenaka, H. Takeshita, H. Hayashi, and Y. Tezuka, Polymer, 42, 3233 (2001). 